\title{
ON QUASI-CONVERGENCE OF SERIES OF INDEPENDENT RANDOM VARIABLES ${ }^{1}$
}

\author{
HOWARD G. TUCKER
}

1. Statement of the result. The purpose of this paper is to prove the following theorem.

THEOREM. Let $\left\{X_{n}\right\}$ be a sequence of independent random variables. $A$ necessary and sufficient condition that there exists a sequence of real numbers $\left\{\lambda_{n}\right\}$ such that $\sum_{n=1}^{\infty}\left(X_{n}-\lambda_{n}\right)$ converges a.s. is that there exist real numbers $\left\{a_{i j}, i, j=1,2, \cdots\right\}$ such that

(i) $a_{i j} \rightarrow 1$ as $i \rightarrow \infty$, for every $j$,

(ii) $S_{i}=\sum_{j=1}^{\infty} a_{i j} X_{j}$ converges a.s., for every $i$, and

(iii) $S_{i} \rightarrow$ (some random variable) $S$ a.s. as $i \rightarrow \infty$.

The sufficient part of this theorem was proved by J. Marcinkiewicz and A. Zygmund [3]. An entirely different proof of this is given here by means of concentration functions and, in particular, by using a theorem due to $\mathrm{K}$. Ito $([1, \mathrm{p} .46]$, and restated and proved in a different manner in [4]). The statement and proof that the condition given in the theorem is necessary appears to be new. In \$2 a lemma is proved which is used to prove that the condition is sufficient; besides its use in this paper this lemma should find considerable application. In $\$ 3$ the theorem is proved. It should be pointed out that no use is made of the independence of the random variables in the proof that the condition is necessary.

2. A lemma. A particular case (in a sense) of the lemma given in this section was stated but not proved by P. Lévy [2, p. 134] and was stated and proved in [4] (see Lemma 4, pp. 719, 720). Since this particular case is not general enough to use in the proof that the condition given in the theorem is sufficient, a more general lemma must be stated and proved.

Let us recall that if $F$ is a probability distribution function, then its concentration function $Q_{F}$ is defined by

$$
Q_{F}(L)=\sup _{x}\{F(x+L+0)-F(x-0)\},
$$

for all $L \geqq 0$. For some of the known properties of concentration functions the reader is referred to [2] and [4].

Received by the editors December 19, 1963.

1 This research was supported in part by the National Science Foundation, Grant No. GP-1365. 
Lemma. Let $F, F_{1}, F_{2}, \cdots$ be a sequence of distribution functions, and let $Q, Q_{1}, Q_{2}, \cdots$ be the corresponding concentration functions. If $F_{n}(x) \rightarrow F(x)$ as $n \rightarrow \infty$ at all $x$ at which $F$ is continuous, then $Q_{n}(x)$ $\rightarrow Q(x)$ as $n \rightarrow \infty$ at all $x$ at which $Q$ is continuous.

Proof. Let $L$ be a point at which $Q$ is continuous. It suffices to prove that

$$
\limsup _{n \rightarrow \infty} Q_{n}(L) \leqq Q(L) \quad \text { if } L \geqq 0
$$

and

$$
\lim _{n \rightarrow \infty} Q_{n}(L) \geqq Q(L) \quad \text { if } L>0 .
$$

In order to prove (1) it is enough to show that any sequence $\left\{n^{\prime}\right\}$ of positive integers contains a subsequence $\left\{n^{\prime \prime}\right\}$ such that $\lim \sup _{n^{\prime \prime} \rightarrow \infty} Q_{n^{\prime \prime}}(L) \leqq Q(L)$. Let $P_{n}$ and $P$ denote the LebesgueStieltjes measures determined by $F_{n}$ and $F$ respectively. For every $n$ let $x_{n}$ be chosen such that

$$
Q_{n}(L) \leqq P_{n}\left[x_{n}, x_{n}+L\right]+1 / n .
$$

Given $\left\{n^{\prime}\right\}$, let $\left\{n^{\prime \prime}\right\} \subset\left\{n^{\prime}\right\}$ be such that $x_{n^{\prime \prime}} \rightarrow$ (some) $x$. If $x=\infty$ or $-\infty$, then by hypothesis, $P_{n^{\prime \prime}}\left[x_{n^{\prime \prime}}, x_{n^{\prime \prime}}+L\right] \rightarrow 0$ as $n^{\prime \prime} \rightarrow 0$, thus verifying the inequality. If $x$ is finite, then, for arbitrary $\epsilon>0$,

$$
\limsup _{n^{\prime \prime} \rightarrow \infty} P_{n^{\prime \prime}}\left[x_{n^{\prime \prime}}, x_{n^{\prime \prime}}+L\right] \leqq P[x-\epsilon, x+L+\epsilon] \leqq Q(L+2 \epsilon),
$$

and since $Q$ is continuous at $L$, this inequality establishes (1). To prove (2), let $\epsilon>0$ be arbitrary, and let $0<L_{1}<L$ be such that $Q\left(L_{1}\right)$ $>Q(L)-\epsilon$ (since $L>0$ is a continuity point of $Q$ ). Let $x$ be such that $P\left[x, x+L_{1}\right]>Q\left(L_{1}\right)-\epsilon$ and select $\delta>0$ such that $x-\delta$ and $x+L_{1}+\delta$ are continuity points of $F$ and $L_{1}+2 \delta<L$. Then

$$
\begin{aligned}
Q(L)-2 \epsilon & <Q\left(L_{1}\right)-\epsilon<P\left[x, x+L_{1}\right] \\
& \leqq P\left[x-\delta, x+L_{1}+\delta\right]=\lim _{n \rightarrow \infty} P_{n}\left[x-\delta, x+L_{1}+\delta\right] \\
& \leqq \liminf _{n \rightarrow \infty} Q_{n}\left(L_{1}+2 \delta\right) \leqq \liminf _{n \rightarrow \infty} Q_{n}(L),
\end{aligned}
$$

which, by the arbitrariness of $\epsilon$, proves (2) and concludes the proof of the lemma.

3. Proof of the theorem. We first prove that the condition is sufficient. For $L \geqq 0$, let $Q_{i, n}(L)$ denote the concentration function of $\sum_{j=1}^{n} a_{i j} X_{j}$, let $Q_{i, \infty}(L)$ denote the concentration function of $S_{i}$, let 
$Q_{\infty, n}(L)$ denote the concentration function of $\sum_{i=1}^{n} X_{i}$, and let $Q(L)$ denote the concentration function of $S$. (Note: $L>0$ and $S$ being finite with positive probability imply $Q(L)>0$.) Let $L>0$ be a continuity point of $Q, Q_{\infty, n}$, and $Q_{n, \infty}, n=1,2, \cdots$. Then the lemma and (iii) imply $Q_{i, \infty}(L) \rightarrow Q(L)>0$ as $i \rightarrow \infty$. For $\epsilon>0,0<2 \epsilon<Q(L)$, we have

$$
Q_{i, \infty}(L)>Q(L)-\epsilon
$$

for all $i>$ (some) $i_{0}$. Take a fixed value of $n$. Then (i) implies that $\sum_{j=1}^{n} a_{i j} X_{j} \rightarrow \sum_{j=1}^{n} X_{j}$ a.s. as $i \rightarrow \infty$, which in turn implies by the lemma that $Q_{i, n}(L) \rightarrow Q_{\infty, n}(L)$ as $i \rightarrow \infty$, or

$$
Q_{\infty, n}(L)>Q_{i, n}(L)-\epsilon
$$

for all $i>$ (some) $i_{1}$. By Lemma 2 in $[4], Q_{i, n}(L) \geqq Q_{i, \infty}(L)$, and hence by (4), we have

$$
Q_{\infty, n}(L)>Q_{i, \infty}(L)-\epsilon
$$

for all $i>i_{1}$. For $i>\max \left\{i_{0}, i_{1}\right\}$, we obtain, by (3) and (5), the inequality

$$
Q_{\infty, n}(L)>Q(L)-2 \epsilon .
$$

Since (by Lemma 2 in $[4]$ ), $\left\{Q_{\infty, n}(L)\right\}$ is a nonincreasing sequence (in $n$ ), then

$$
\lim _{n \rightarrow \infty} Q_{\infty, n}(L)>Q(L)-2 \epsilon>0 .
$$

Inequality (6) and the theorem of K. Ito (p. 720 in [4]) then imply the sufficiency of the condition.

We now prove that the condition is necessary. Let $\left\{\lambda_{n}\right\}$ be a sequence of real numbers such that $\sum_{n=1}^{\infty}\left(X_{n}-\lambda_{n}\right)$ converges a.s.; we shall prove that there exist real numbers $\left\{a_{i j}\right\}$ such that (i), (ii) and (iii) are true. We assume that $\sum_{n=1}^{\infty} X_{n}$ does not converge a.s., i.e., $\sum_{n=1}^{\infty} \lambda_{n}$ diverges; otherwise $a_{i j}=1$ for all $i$ and $j$. The proof is broken up into three cases.

Case 1. Suppose the sequence $\left\{\lambda_{n}\right\}$ is unbounded. Then for every positive integer $m$ there is an integer $n_{m}>m$ such that $\left|\sum_{k=1}^{m} \lambda_{k} / \lambda_{n_{m}}\right|$ $\leqq 1$. Now define

$$
a_{m j}=\left\{\begin{array}{l}
1 \text { if } 1 \leqq j \leqq m, \\
-\sum_{k=1}^{m} \lambda_{k} / \lambda_{n_{m}} \quad \text { if } j=n_{m}, \\
0 \quad \text { if } j>m, j \neq n_{m} .
\end{array}\right.
$$


Then

$$
\begin{aligned}
S_{m} & =X_{1}+\cdots+X_{m}-\left(\sum_{k=1}^{m} \lambda_{k} / \lambda_{n_{m}}\right) X_{n_{m}} \\
& =\left(X_{1}-\lambda_{1}\right)+\cdots+\left(X_{m}-\lambda_{m}\right)-\left(\sum_{k=1}^{m} \lambda_{k} / \lambda_{n_{m}}\right)\left(X_{n_{m}}-\lambda_{n_{m}}\right) .
\end{aligned}
$$

Since $X_{n_{m}}-\lambda_{n_{m}} \rightarrow 0$ a.s. as $m \rightarrow \infty$, and since its coefficient in the last expression is bounded, then $S_{m} \rightarrow \sum_{n=1}^{\infty}\left(X_{n}-\lambda_{n}\right)$ a.s. as $m \rightarrow \infty$. Thus (ii) and (iii) are shown to hold, and (i) is trivially true.

Case 2. Suppose $\left\{\lambda_{n}\right\}$ are bounded, but suppose there exists an increasing sequence of integers $0=k_{0}<k_{1}<\cdots$ such that if $\beta_{n}$ $=\sum_{j=k_{n-1}+1}^{\boldsymbol{k}_{n}} \lambda_{j}$, then the $\left\{\beta_{n}\right\}$ are unbounded. Let us denote $Y_{n}$ $=\sum_{j=k_{n-1}+1}^{k_{n}} X_{j}$. (The $Y_{n}$ 's are independent, but no use will be made of this fact.) Now $\sum_{n=1}^{\infty}\left(Y_{n}-\beta_{n}\right)$ converges a.s. and the $\left\{\beta_{n}\right\}$ are unbounded. Thus Case 1 applies, and there exist real numbers $\left\{a_{m n}^{\prime}\right\}$ such that $a_{m n}^{\prime} \rightarrow 1$ as $m \rightarrow \infty$ for each $n, S_{m}^{\prime}=\sum_{n=1}^{\infty} a_{m n}^{\prime} Y_{n}$ converges a.s., and $S_{m}^{\prime} \rightarrow S$ a.s. as $m \rightarrow \infty$. Let $a_{m n}=a_{m r}^{\prime}$ if $k_{r-1}+1 \leqq n \leqq k_{r}$. Then the same $S_{m}^{\prime}=\sum_{n=1}^{\infty} a_{m n} X_{n}$ converges a.s. and $S_{m}^{\prime} \rightarrow S$ a.s.

Case 3. Suppose the $\left\{\lambda_{n}\right\}$ are bounded but that there exists no increasing sequence of integers $\left\{k_{n}\right\}$ such that $\left\{\beta_{n}\right\}$ are unbounded, where $\beta_{n}$ is defined in Case 2. We denote $\gamma_{n}=\lambda_{1}+\cdots+\lambda_{n}$.

We first prove that the $\left\{\gamma_{n}\right\}$ are bounded. Suppose this were not so; suppose $\left\{\gamma_{n}\right\}$ are unbounded above. Then there exists a $k_{1}$ such that $\gamma_{k_{1}}=\lambda_{1}+\lambda_{2}+\cdots+\lambda_{k_{1}}=\beta_{1}>1$. In general, there is a $k_{n}$ such that $\gamma_{k_{n}}=\lambda_{1}+\cdots+\lambda_{k_{n}}=\beta_{n}+\gamma_{k_{n-1}}>\gamma_{k_{n-1}}+n$, or $\beta_{n}>n$. Hence there exists an increasing sequence of integers $\left\{k_{n}\right\}$ such that $\left\{\beta_{n}\right\}$ are unbounded, which contradicts the hypothesis of Case 3. Thus $\left\{\gamma_{n}\right\}$ are bounded.

Since $\left\{\gamma_{n}\right\}$ are bounded, there are limit points of it. There are at least two limit points of $\left\{\gamma_{n}\right\}$, for, otherwise, this sequence would converge, which would contradict our hypothesis. Without loss of generality we may suppose 0 to be the smallest limit point and $a>0$ the largest. Let $\epsilon>0$ be such that $3 \epsilon<a$. Then there exists an increasing sequence of positive integers $\left\{k_{n}\right\}$ such that $a-\epsilon<\gamma_{k_{2 n-1}}<a+\epsilon$ and $-\epsilon<\gamma_{k_{2 n}}<\epsilon$ for $n=1,2, \cdots$, and $-\epsilon<\gamma_{n}<a+\epsilon$ for all $n \geqq k_{1}$. Now let

$$
\begin{aligned}
a_{m, 1} & =a_{m, 2}=\cdots=a_{m, k_{m}}=1, \\
a_{m, k_{m}+1} & =a_{m, k_{m}+2}=\cdots=a_{m, k_{m+1}}=K_{m}, \quad a_{m, j}=0 \text { if } j>k_{m+1}
\end{aligned}
$$

where 


$$
K_{m}=-\sum_{j=1}^{k_{m}} \lambda_{j} / \sum_{j=k_{m}+1}^{k_{m+1}} \lambda_{j}
$$

Then

$$
\begin{aligned}
S_{m} & =\sum_{j=1}^{k_{m}} X_{j}+K_{m} \sum_{j=k_{m}+1}^{k_{m+1}} X_{j} \\
& =\sum_{j=1}^{k_{m}}\left(X_{j}-\lambda_{j}\right)+K_{m} \sum_{j=k_{m}+1}^{k_{m+1}}\left(X_{j}-\lambda_{j}\right) .
\end{aligned}
$$

Since $\left\{K_{m}\right\}$ is bounded, i.e.,

$$
\left|K_{m}\right| \leqq\left(\sum_{j=1}^{k_{1}} \lambda_{j}+2 \epsilon+a\right) /(a-2 \epsilon),
$$

it follows that $S_{m} \rightarrow \sum_{n=1}^{\infty}\left(X_{n}-\lambda_{n}\right)$ a.s. as $m \rightarrow \infty$, which concludes the proof that the condition is necessary.

4. Acknowledgements. Professor J.-P. Kahane suggested this study, and he shortened considerably my original proof of Case 3 in the proof that the condition is necessary. The referee suggested the present more readable proof of the lemma in $\$ 2$. To these two men I wish to express my gratitude.

\section{REFERENCES}

1. K. Ito, Stochastic processes, Part I (in Russian), Publishers of Foreign Literature, Moscow, 1960.

2. P. Lévy, Theorie de l'addition des variables aléatories, Gauthier-Villars, Paris, 1937.

3. J. Marcinkiewicz and A. Zygmund, Quelques thêorèmes sur les fonctions indépendentes, Studia Math. 7 (1938), 104-120.

4. H. G. Tucker, Quasi-convergent series of independent random variables, Amer. Math. Monthly 70 (1963), 718-722.

University of California, Riverside, and The Institute for Advanced Study 